\title{
Improved silicon nitride surfaces for next-generation
}

\section{microarrays}

Jonathan G. Terry, ${ }^{* 1}$ Colin J. Campbell, ${ }^{* 2}$ Alan J. Ross, ${ }^{2}$ Andrew D. Livingston, ${ }^{2}$ Amy H. Buck, ${ }^{2}$

Paul Dickinson, ${ }^{2}$ Christopher P. Mountford, ${ }^{3}$ Stuart A.G. Evans, ${ }^{4}$ Andrew R. Mount, ${ }^{4}$ John S. Beattie, ${ }^{2}$

Jason Crain, ${ }^{3}$ Peter Ghazal, ${ }^{2}$ Anthony J. Walton. ${ }^{1}$

1 Institute for Integrated Micro and Nano Systems, University of Edinburgh,

Scottish Microelectronics Centre, West Mains Road, Edinburgh, EH9 3JF, UK

2 Scottish Centre for Genomic Technology and Informatics, University of Edinburgh,

The Chancellor's Building, 49 Little France Crescent, Edinburgh, EH16 4SB, UK

3 School of Physics, University of Edinburgh, James Clerk Maxwell Building, Mayfield Road, Edinburgh, EH9 3JZ, UK.

4 School of Chemistry, University of Edinburgh, Joseph Black Building,

West Mains Road, Edinburgh, EH9 3JJ, UK.

*Corresponding Authors: $\quad$ Jon.Terry@ed.ac.uk

Colin.Campbell@ed.ac.uk 
Probe sequences for microarray fabrication

\begin{tabular}{|c|c|c|c|c|}
\hline Probe & & Description & Probe ID & Oligo Sequence \\
\hline vMC105 & neg1 & $\mathrm{m} 107$ & 35993 & $\begin{array}{l}\text { ACGCCGTATACTTACACGTGCGTAACGA } \\
\text { GGCGGAAGACCCACATTTCGGGCGGTT }\end{array}$ \\
\hline vMC148 & neg2 & $\mathrm{m} 144$ & 36036 & $\begin{array}{l}\text { GTAACGGGCTCTACCAAAAACATATCGA } \\
\text { CGTCTATGTGGACGGAGGCTTGGAGCA }\end{array}$ \\
\hline vMC149 & neg3 & $\mathrm{m} 145$ & 36037 & $\begin{array}{l}\text { CCAAGACCGCGACCGTTGAGGTCTCTAC } \\
\text { GAACATCGAGGTCTCGGAAACCACGTC }\end{array}$ \\
\hline vMC153 & neg4 & $\mathrm{m} 149$ & 36041 & $\begin{array}{l}\text { GAAGACATAGCGTGCGCTGATCTGTCTG } \\
\text { TGCCGCGTCGGAGTCAGCTCGTCCAGA }\end{array}$ \\
\hline vMC164 & neg5 & $\mathrm{m} 160$ & 36052 & $\begin{array}{l}\text { CGCAACCGAACGCGGACCGGACAAACG } \\
\text { CTCGGAGTACAGATGTCATGCAGACCAC }\end{array}$ \\
\hline cMU001 & pos1 & AB027565 & 36284 & $\begin{array}{l}\text { CTGGCCCTGCAGCTTCTTTTCTTGCCCT } \\
\text { CCCCTTTGCCTGTCCCCACCTGCAGTA }\end{array}$ \\
\hline cMU010 & pos2 & D85137 & 36249 & $\begin{array}{l}\text { CTGCCGCCAGCCCTGCCCGACTATCAT } \\
\text { GAAGCACACTCCTTCCCACTGCACATTT }\end{array}$ \\
\hline cMU025 & pos3 & NM_008608 & 36276 & $\begin{array}{l}\text { GCGTTCGCTGCTGGACAAGGTCTGACC } \\
\text { CCCACCACTGGCCCACCCGCTTCTACCA }\end{array}$ \\
\hline cMU012 & pos4 & U47325 & 36250 & $\begin{array}{l}\text { ACCCTATCCCTGCACTGCCCTGTGTTCC } \\
\text { CTTCCATAGCCAACCTTGCTGCTCCAG }\end{array}$ \\
\hline cMU034 & pos5 & X03672 & 36260 & $\begin{array}{l}\text { AAGTGGTTACAGGAAGTCCCTCACCCTC } \\
\text { CCAAAAGCCACCCCCACTCCTAAGAGG }\end{array}$ \\
\hline vMC109 & test1 & M114 & 35997 & $\begin{array}{l}\text { GAAACATCTGATCCTCCGATCCAGCCAC } \\
\text { CCGTCCCCACGCGCTCAAGGAGCACGC }\end{array}$ \\
\hline vMC124 & test2 & $\mathrm{m} 123 \mathrm{ex} 4$ & 36012 & $\begin{array}{l}\text { CTCCCCAGGCAATGAACAATCAGTCTTG } \\
\text { GCCCATGCGGCACGCTCATCTAGTGCG }\end{array}$ \\
\hline vMC139 & test3 & $\mathrm{m} 135$ & 36027 & $\begin{array}{l}\text { ATTAACACCACAGAATGTCCCTCTCCCT } \\
\text { CTGAGACCGTGTCCGCTCAGACCATCG }\end{array}$ \\
\hline vMC180 & test4 & $\mathrm{m} 163$ & 36068 & $\begin{array}{l}\text { AGAAGGCCATCTGAAGAACGATTCCCA } \\
\text { CCAGCACCACCCACCCGACGCCCCAACC }\end{array}$ \\
\hline vMC186 & test5 & m02as & 36074 & $\begin{array}{l}\text { GTACTTTGGGTGGGACGGCGGGTGCTT } \\
\text { TGGGCGGTGGGACGTACGGAACGACCTT }\end{array}$ \\
\hline
\end{tabular}

Probes listed as "test genes" are viral oligonucleotides. Viral oligos were designed against all 170 open reading frames (ORFs) of murine cytomegalovirus as annotated by Rawlinson et al (1). Positive control probes are oliognucleotides designed against murine cellular genes pre-selected from published house-keeping genes. ${ }^{\mathrm{i}}$ Oligonucleotide probes were designed using Oligo 6 primer design software (Molecular Biology Insights, http://www.oligo.net/contact.htm). All probes were synthesized by MWG-Biotech. 
${ }^{\mathrm{i}}$ Rawlinson, W., H. Farrell, and B. Barrell. 1996. Analysis of the complete DNA sequence of murine cytomegalovirus. J Virol. 70:8833-49. 